\title{
DEGRADAÇÃO AMBIENTAL E CRESCIMENTO ECONÔMICO: A CURVA DE KUZNETS AMBIENTAL PARA O CERRADO ${ }^{1}$
}

\author{
Maria Virgínia da Silva Colusso² \\ José Luiz Parré3 \\ Eduardo Simões Almeida ${ }^{4}$
}

RESUMO: Este estudo tem como objetivo verificar a existência de relação entre degradação ambiental e crescimento econômico, sob a hipótese da Curva de Kuznets Ambiental, para o Cerrado brasileiro, no ano de 2008. Definiu-se como variável dependente a área desmatada de Cerrado dos municípios do bioma e, como variáveis explicativas, o PIB per capita e sua forma quadrática e cúbica, a densidade demográfica, a área plantada e o efetivo dos rebanhos bovinos. Os efeitos espaciais foram considerados na análise. Os resultados apontam que, num primeiro momento, o crescimento da renda per capita ajuda a diminuir o desmatamento na região; porém, o aumento continuado desta renda faz com que o desmatamento volte a crescer.

Palavras-chave: Curva de Kuznets Ambiental, desmatamento, econometria espacial.

ABSTRACT: This paper aims to verify the existence of relationship between environmental degradation and economic growth, based on the Environmental Kuznets Curve hypothesis for the Brazilian Cerrado in 2008. The deforested area of the Cerrado's municipalities was defined as dependent variable and as explanatory variables the GDP per capita and its quadratic and cubic form, population density, the planted area and the cattle. Spatial econometric methods were used. The results show that, initially, the growth of per capita income helps to reduce deforestation in the region. However, the continuous increase of per capita GDP leads to deforestation to grow back.

Keywords: deforestation, Environmental Kuznets Curve, spatial econometrics.

1 Recebido em: 03/07/2013. Aceito em: 05/09/2013.

2 Economista (UFSM); Mestre em Economia (PCE/UEM).

E-mail: virginia.colusso@gmail.com

3 Professor Associado do Departamento de Economia da Universidade Estadual de Maringá (DCO/PCE/UEM); Bolsista de Produtividade em Pesquisa do CNPq.

E-mail: jlparre@uem.br

4 Professor Adjunto do Departamento de Economia da Universidade Federal de Juiz de Fora (CMEA/UFJF).E-mail: eduardo.almeida@ufjf.edu.br 


\section{Introdução}

O Cerrado é o segundo maior bioma da América do Sul, sendo superado apenas pelo bioma da Amazônia. Apesar da abundância hídrica e de ser a savana mais rica em diversidade biológica do mundo, tal bioma sofre com as profundas ações antrópicas, grande parte em decorrência da expansão da fronteira agrícola. Da vastidão de sua área de $2.036 .448 \mathrm{~km}^{2}$, equivalente a quase $24 \%$ do território nacional, estima-se que $47,84 \%$ do seu território tenha sido desmatado até o ano de 2008 , e apenas $7,44 \%$ seja protegido por unidades de conservação.

Apesar de se saber que muitos dos problemas ambientais são oriundos da atividade econômica, acredita-se que ela também possa engendrar soluções. Assim, a Curva de Kuznets Ambiental (CKA) surge como uma conjectura ao questionamento: Can economic growth be part of the solution rather than the cause of environmental problems? (ROTHMAN; DE BRUYN, 1998, p. 143).

Em alusão ao artigo de Simon Kuznets, de 1966, que relacionava desigualdade entre distribuição e nível de renda per capita, a Curva de Kuznets Ambiental relaciona degradação ambiental com nível de renda per capita. Sob a conjectura de que o incremento da produção resultaria em maior pressão ao meio ambiente, num primeiro momento a prioridade de um país seria o crescimento econômico. Posteriormente, tendo atingido determinado nível de desenvolvimento, suas preferências mudariam e com isso se passaria a exercer maior controle da qualidade da natureza, reduzindo a degradação ao meio ambiente. Essa transição seria representada graficamente por uma parábola com concavidade voltada para baixo, ou curva em forma de "U" invertido, característica da CKA.

A proposta desse estudo é dar continuidade à pesquisa acerca do tema, tendo como objetivo principal verificar a existência de relação entre degradação ambiental e crescimento econômico, sob a hipótese da Curva de Kuznets Ambiental, para o Cerrado brasileiro, no ano de 2008. 
O trabalho está disposto em cinco seções, sendo a primeira delas esta introdução. A seção 2 trata da revisão da literatura e de expostos teóricos sobre a CKA. A seção 3 apresenta o modelo e a metodologia de estimação. A seção 4 apresenta os resultados obtidos, bem como sua análise e interpretação. Por fim, a seção 5 conclui o trabalho.

\section{A curva de kuznets ambiental}

Os trabalhos empíricos acerca da relação entre degradação ambiental e crescimento econômico remontam ao pioneiro artigo de Grossman e Krueger, de 1991, intitulado "Environmental Impacts of a North American Free Trade Agreement". A motivação dos autores foram os questionamentos de grupos ambientalistas sobre as pressões ambientais após a formação do bloco de livre comércio, NAFTA, uma vez que, com a abertura comercial entre os países membros, a produção se expandiria. Os autores reconhecem que alguns poluentes são subprodutos da atividade econômica e que, dada sua expansão, a tendência é a emissão desses poluentes também aumentar, mas, por outro lado: "as a society becomes richer its members may intensify their demands for a more healthy and sustainable environment, in which case the government may be called upon to impose more stringent environmental controls. " (GROSSMAN; KRUEGER, 1991, p. 7)

Grossman e Krueger (1991) relacionam a qualidade do ar a três poluentes atmosféricos $-\mathrm{SO}_{2}$, partículas em suspensão e matéria escura - com o crescimento econômico, representado pelo PIB per capita em suas formas quadrática e cúbica. Os resultados indicam a presença de relação do tipo Curva de Kuznets Ambiental (posteriormente assim denominada) nas análises feitas, isto é, aumentava-se a emissão de poluentes atmosféricos até o ponto máximo em que o contínuo crescimento econômico os fazia decrescer, configurando, assim, a curva em forma de " $U$ " invertido. 
Mueller (2007, p.62) define a hipótese da Curva de Kuznets Ambiental:

Em um país subdesenvolvido, cuja renda per capita aumenta consistentemente, um emprego de quantidades crescentes de energia e materiais conduz a uma degradação ambiental (...) cada vez maior. Mas isso aconteceria até certo nível de renda per capita. Se a $Y / P$ [razão entre produto $Y$ e a população $P$, resultando na renda per capita] desse país continuar a crescer, cedo ou tarde será atingido um nível de renda per capita após o qual aumentos ulteriores nesse indicador de desenvolvimento trariam reduções na magnitude dos indicadores de degradação ambiental.

A CKA seria resultado de três efeitos, de acordo com Borghesi (2002, apud MUELLER, 2007): escala, composição e mudança tecnológica. Quanto maior a escala de produção de uma economia, maior é também a emissão de resíduos poluentes, sendo este efeito predominante em estágios iniciais de desenvolvimento. O efeito composição refere-se à estrutura do processo produtivo: uma economia cujo PIB é oriundo em sua maior parte do setor de serviços poderia ser considerada mais "limpa". Ainda, o efeito composição agiria no sentido inverso ao efeito escala. O efeito mudança tecnológica é a intenção de progredir para tecnologias mais eficientes e mais "limpas" também. Em níveis mais elevados de desenvolvimento, seriam atribuídos pesos maiores aos efeitos composição e mudança tecnológica.

Quanto ao formato característico da curva, Carvalho e Almeida (2010) apontam algumas possíveis causas para a transição de uma ascendência 
positiva para uma inclinação negativa tais como elasticidade-renda positiva para qualidade ambiental, alterações na composição da produção e do consumo, aumento da difusão da educação ambiental e conscientização da população, maior rigor da regulação ambiental e incremento tecnológico.

Rothman e de Bruyn (1998) afirmam que as evidências da existência de tal relação acabaram por gerar interpretações equivocadas de que somente mediante crescimento econômico é que políticas ambientais efetivas poderiam ser implementadas:

The presumption of income determinism hampers a fuller understanding of the factors underlying the changes in environmentincome relationships and can result in naive conclusions about the role of economic growth in reducing environmental pressure (ROTHMAN; DE BRUYN, 1998, p. 144).

Desse modo, os autores mostram que o modo mais propício de se ver a CKA é como uma hipótese e não como relação determinística. Com isso, concluem que não se pode dizer que a hipótese foi confirmada ou rejeitada e que os estudos empíricos sobre ela apenas mostram diferentes abordagens, deficiências e sugestões de como superar tais faltas.

\section{Metodologia}

Para a economia ecológica, a localização geográfica é um fator bastante relevante, uma vez que a distribuição geográfica interfere no fenômeno em análise. Assim, optou-se por utilizar o instrumental da econometria espacial, uma vez que métodos econométricos "tradicionais" seriam 
limitados quanto à capacidade de controlar tais efeitos espaciais. Anselin (1999, p. 1) define econometria espacial: spatial econometrics is a subfield of econometrics that deals with the treatment of spatial interaction (spatial autocorrelation) and spatial structure (spatial heterogeneity) in regression models.

LeSage (1998) ressalva que observações mais próximas entre si apresentam maior grau de dependência espacial (teorema da ciência regional), e expõe assim o conceito de vizinhança na econometria espacial. Nesse sentido, a contiguidade, isto é, a posição relativa da observação no espaço em relação às demais observações, permite determinar quem são considerados vizinhos. Uma maneira de quantificar tal contiguidade pode ser por meio da atribuição de um peso espacial. Define-se então um arranjo para as interações espaciais, conhecido como matriz de pesos espaciais, tornando possíveis a estimação de parâmetros e a verificação do grau de interação entre as variáveis em análise. A matriz pode ser baseada na contiguidade (com convenção do tipo rainhaqueen - ou torre - rook), na distância (ou a inversa dela), em um número determinado $k$ de vizinhos, entre outros, sendo sua escolha compatível com as características do fenômeno em estudo.

Como auxílio na identificação do modelo econométrico espacial apropriado, faz-se a Análise Exploratória de Dados Espaciais (AEDE). Trata-se de um instrumental de análise estatística de informações geográficas que permite inferir padrões espaciais nos dados e a dedução de hipóteses. A AEDE é mais apropriada para a análise de variáveis espacialmente densas, isto é, dividindo-as por algum indicador de intensidade, como população ou área.

Assim, distinguem-se dois efeitos espaciais: a dependência (ou autocorrelação espacial) e a heterogeneidade espacial. Na definição mais simplificada de Anselin (1999, p. 4), dependência especial é: "the coincidence of value similarity with location similarity”. A ocorrência da heterogeneidade espacial se dá devido ao fato de fenômenos espaciais não apresentarem estabilidade estrutural (como coeficientes variáveis, variância não constante ou ainda formas funcionais diferentes para 
determinados subconjuntos de dados). Consequentemente, dificultase o ajustamento de um mesmo modelo teórico para todo e qualquer conjunto de dados, acarretando perda de eficiência ou até mesmo estimativas viesadas. Na definição de LeSage (1998, p. 6): the term spatial heterogeneity refers to variation in relationships over space. In the most general case consider that we might expect a different relationship to hold for every point in space.

Para averiguar a existência de autocorrelação espacial, algumas estatísticas de teste cumprem essa função, como as estatísticas $I$ de Moran, $c$ de Geary e $G$ de Getis-Ord. Optou-se por utilizar, nesse estudo, apenas a estatística $I$ de Moran, por ser ela a medida mais usual, não se incorrendo assim em prejuízo para os resultados obtidos. A estatística de teste supõe como hipótese nula a aleatoriedade espacial, isto é, que o fenômeno em estudo não dependeria da sua localização.

Visto que tanto a ausência quanto à forte presença de autocorrelação global podem ocultar associações espaciais locais, como clusters ou outliers espaciais, Anselin (1995) propôs um novo indicador capaz de capturar padrões locais de associação linear, o de $I$ de Moran local. Dessa forma, o $I$ de Moran local fornece indícios do grau de agrupamento dos valores análogos em torno de uma determinada observação da amostra, identificando clusters espaciais estatisticamente significantes.

Assim como se calcula o $I$ de Moran global multivariado, é possível calcular também o $I$ de Moran local multivariado a fim de se obter o grau de associação linear entre uma variável em uma dada localidade e a média da outra variável em regiões vizinhas. De acordo com a dispersão dos valores obtidos de Moran, pode-se verificar a formação de clusters ou agrupamentos espaciais tanto para a estatística $I$ de Moran univariada quanto para a multivariada. São definidos quatro tipos desses agrupamentos: Alto-Alto (AA), Baixo-Baixo (BB), Alto-Baixo (AB) e Baixo-Alto (BA). Um relevante problema sobre o mapa de dispersão de Moran é que ele exibe tanto clusters estatisticamente significantes quanto os que não o são. A solução apresentada para este problema seria combinar o mapa de dispersão de Moran com o mapa de significância 
dos valores obtidos de associação local, apresentando, assim, somente clusters estatisticamente significantes.

\section{Modelos econométricos espaciais}

A proposta é estimar o modelo da CKA utilizando seis diferentes métodos: (0) modelo sem correção para dependência espacial (estimação por Mínimos Quadrados Ordinários); (1) modelo de defasagem espacial (SAR); (2) modelo de erro espacial (SEM); (3) modelo regressivo cruzado espacial (SLX); (4) modelo de Durbin espacial (SDM); e (5) modelo de Durbin espacial do erro (SDEM).

O modelo de defasagem espacial ou Spatial Autoregressive Model (SAR) incorpora a variável dependente defasada espacialmente a fim de capturar o efeito de "vizinhança" do fenômeno em estudo. O modelo pode ser expresso por:

$$
y=\rho W y+X \beta+\varepsilon
$$

em que $\rho$ é o coeficiente autorregressivo espacial (um escalar); Wy é o vetor $n \times 1$ representando a defasagem espacial da variável dependente; $X$ é a matriz $\mathrm{n} \times \mathrm{k}$ de variáveis explicativas; $\beta$ é o vetor $\mathrm{k} \times 1$ de coeficientes de regressão; e $\varepsilon$ é o vetor $n \times 1$ de erros aleatórios.

O modelo com erro autorregressivo espacial expressa no termo de erro aqueles efeitos que não podem ser modelados. Dessa forma, a autocorrelação espacial nesse modelo se apresenta apenas no termo de erro da regressão. O significado intuitivo desse modelo é que o padrão espacial manifestado no termo de erro é dado por efeitos não modelados por conta da falta de adequada medida, que, por sua vez, estão espacialmente autocorrelacionados, mas não estão correlacionados com nenhuma variável explicativa inserida no modelo de regressão. Assim, o modelo pode ser expresso da seguinte forma:

$$
y=X \beta+\lambda W u+\varepsilon
$$


em que o parâmetro do erro autorregressivo espacial é restringido como $|\lambda|<1$ e acompanha a defasagem espacial do termo de erro $(W u)$.

No modelo regressivo cruzado espacial, supõe-se que algumas ou até mesmo todas as variáveis explicativas tenham seus efeitos espraiados para regiões vizinhas, além de apresentar uma defasagem espacial para a variável dependente. $\mathrm{O}$ modelo é expresso por:

$$
y=X \beta+W X \tau+\varepsilon
$$

em que $\tau$ é um vetor de coeficientes espaciais que acompanha as defasagens espaciais das variáveis explicativas $(W X)$, que permitem denotar os efeitos de transbordamento localizados.

O modelo de Durbin espacial incorpora a ideia de transbordamento pela defasagem das variáveis independentes (WX) e também pela inclusão de uma variável endógena defasada espacialmente (Wy). Este modelo pode ser especificado como:

$$
y=\lambda W y+X \beta-\lambda W X \beta+\varepsilon
$$

O modelo misto com defasagem espacial e erro autorregressivo espacial incorpora tanto o coeficiente de defasagem espacial $(\rho)$ quanto parâmetro do termo de erro autorregressivo $(\lambda)$. O modelo é expresso por:

$$
y=\rho W_{1} y+X \beta+\lambda W_{\mathbf{2}} u+\varepsilon
$$

Ressalta-se que os modelos espaciais de defasagem (SAR), de erro (SDM), Durbin espacial (SEM) e Durbin espacial do erro (SDEM) foram estimados por máxima verossimilhança, e o modelo regressivo cruzado espacial (SLX), por MQO5.

5 Para mais informações técnicas sobre a taxonomia desses modelos econométricoespaciais, consultar LeSage e Pace (2009). 


\section{Modelo empírico}

Na busca por trabalhos sobre o tema, observou-se grande quantidade de artigos sobre a CKA, relacionando o crescimento econômico à emissão de gases poluentes atmosféricos - o que se deve, muito provavelmente, ao fato de essa se tratar da relação original feita no trabalho pioneiro de Grossman e Krueger (1991) - e à pouca quantidade de trabalhos que consideram o desmatamento de florestas como variável de interesse. Tal escassez reforça a necessidade de maior investigação empírica da questão e incentiva a pesquisa do presente estudo a averiguar a relação entre o desmatamento do bioma Cerrado e o crescimento da região em que ele se encontra. Assim, definiu-se como variável dependente a área de Cerrado desmatada dos municípios que compõem o bioma (DESMAT).

Segundo Mueller (2007, p. 38), "a evolução da degradação ambiental gerada por uma sociedade vai depender da dinâmica dos dois componentes da escala da economia (Y): o da sua população $(\mathrm{P})$ e o da sua produção (material) per capita $(\mathrm{Y} / \mathrm{P})$ ". Ainda, "a distribuição de renda molda a demanda, o padrão de consumo, a estrutura produtiva e a natureza dos resíduos lançados no meio ambiente" (MUELLER, 2007, p. 38). Foram utilizados neste estudo o PIB per capita, como medida da distribuição de renda, e a densidade demográfica, uma vez que se acredita que ela seja um forte agravante dos impactos causados sobre o meio ambiente.

A agropecuária é importante fonte de geração de empregos e renda na região, bem como no incremento da produção agrícola do país, de modo geral. Apesar desses pontos positivos, a atividade causa impactos negativos diretos sobre rios e solo, além de redução da biodiversidade e da cobertura vegetal do Cerrado (EMBRAPA CERRADOS, 2008). Assim, variáveis representativas da atividade agropecuária foram incluídas no estudo, como a área plantada total e o efetivo do rebanho bovino.

O Quadro 1 apresenta o resumo das variáveis utilizadas no modelo, sua descrição, o sinal esperado e a fonte dos dados. 
Quadro 1- Apresentação das variáveis do estudo.

\begin{tabular}{|l|l|l|l|}
\hline Variável & Descrição & Sinal & Fonte \\
\hline DESMAT $_{i}$ & Área de Cerrado desmatada. & & Ibama \\
\hline$Y_{i}$ & PIB per capita. & + & $\begin{array}{l}\text { elaboração própria, } \\
\text { com base em dados } \\
\text { do Sidra/IBGE e } \\
\text { Ipeadata. }\end{array}$ \\
\hline$Y_{i}^{2}$ & PIB per capita ao quadrado. & - & $\begin{array}{l}\text { calculado a partir da } \\
\text { variável Y }\end{array}$ \\
\hline$Y_{i}^{3}$ & PIB per capita ao cubo. & + ou nulo & $\begin{array}{l}\text { calculado a partir da } \\
\text { variável Y }\end{array}$ \\
\hline DEMOG & $\begin{array}{l}\text { Densidade demográfica } \\
\text { (razão do número de } \\
\text { habitantes por área total do } \\
\text { município em km }{ }^{2} \text { ) }\end{array}$ & + & $\begin{array}{l}\text { elaboração própria, } \\
\text { com base em dados } \\
\text { do Sidra/IBGE e } \\
\text { Ipeadata. }\end{array}$ \\
\hline AREAPLANT & $\begin{array}{l}\left.\text { Área plantada total (em km }{ }^{2}\right), \\
\text { intensificada pela área total } \\
\text { do município. }\end{array}$ & + & $\begin{array}{l}\text { elaboração própria, } \\
\text { com base em dados } \\
\text { do Sidra/IBGE }\end{array}$ \\
\hline REBANHO & $\begin{array}{l}\text { Rebanho bovino (número de } \\
\text { cabeças), intensificada pela } \\
\text { área total do município. }\end{array}$ & + & $\begin{array}{l}\text { elaboração própria, } \\
\text { com base em dados } \\
\text { do Sidra/IBGE }\end{array}$ \\
\hline
\end{tabular}

Fonte: Elaboração própria.

A amostra utilizada tratou dos 1306 municípios que apresentam área de Cerrado no Brasil, de acordo o Relatório Técnico de Monitoramento do Desmatamento no Bioma Cerrado do Ibama de 2009. Os dados são analisados na forma de cross-section para o ano de 2008 devido à limitação de dados de desmatamento para os municípios do bioma em diferentes períodos, impossibilitando que o estudo fosse mais abrangente sob o aspecto temporal. Deste modo, o modelo econométrico básico para estimar a CKA pode ser descrito como:

$$
\operatorname{DESMAT}_{i}=\alpha_{0}+\alpha_{1} Y_{i}+\alpha_{2} Y_{i}^{2}+Z_{i} \beta+u_{i}
$$


em que $\mathrm{Z}$ é a matriz de dimensão $\mathrm{n} \times \mathrm{k}$ de variáveis explicativas adicionais (variáveis de controle) do modelo e $\beta$, o vetor $\mathrm{k} \times 1$ de coeficientes.

Alguns autores defendem que a CKA não se sustentaria no longo prazo, sendo seu formato de "U" invertido apenas um estágio inicial desta relação. Rapidamente a degradação tornaria a aumentar, mesmo em níveis mais elevados de desenvolvimento econômico, caracterizando o formato da relação entre crescimento e degradação similar ao de um "N" (CARVALHO; ALMEIDA, 2010). Para captar tal efeito, introduz-se a forma cúbica da variável PIB per capita. Assim, caso a estimação do modelo descrito seja estatisticamente significativa, a forma cúbica da variável PIB per capita será incluída na análise e o novo modelo a ser reestimado será apresentado como:

$$
\operatorname{DESMAT}_{i}=\alpha_{0}+\alpha_{1} Y_{i}+\alpha_{2} Y_{i}^{2}+\alpha_{3} Y_{i}^{3}+Z_{i} \beta+u_{i}
$$

De acordo com Carvalho e Almeida (2010), para que a hipótese da CKA tenha a forma de " $U$ " invertido, é condição necessária que o coeficiente estimado $\alpha_{1}$ seja positivo e significativo, o coeficiente $\alpha_{2}$ seja negativo e o coeficiente $\alpha_{3}$ não seja significativamente diferente de zero (uma vez que o contrário ocorra, isso é, o coeficiente $\alpha_{3}$ seja significativo e positivo, evidenciaria uma CKA em forma de "N"). Há ainda a possibilidade de que a relação entre degradação ambiental seja linear, crescente ou decrescente. Porém, nesses casos, não se configuraria tal relação como sendo a Curva de Kuznets Ambiental.

\section{Análise exploratória}

Diversas configurações de matrizes espaciais foram testadas e os resultados obtidos, Tabela 1, indicam que a matriz de pesos espacial com o maior valor encontrado da estatística $I$ de Moran é a matriz de contiguidade do tipo torre. Isto permite inferir que a autocorrelação espacial da variável desmatamento do Cerrado para o ano de 2008 é mais bem captada utilizando-se esta configuração espacial. 
Tabela 1 - Matrizes de pesos espaciais e valores encontrados da estatística $I$ de Moran.

\begin{tabular}{|c|c|c|}
\hline Tipo de matriz & $I$ de Moran & p-value* \\
\hline Rook & 0,4361 & 0,0010 \\
\hline Queen & 0,4227 & 0,0010 \\
\hline 5 vizinhos & 0,3857 & 0,0010 \\
\hline 10 vizinhos & 0,3282 & 0,0010 \\
\hline 15 vizinhos & 0,2969 & 0,0010 \\
\hline
\end{tabular}

*A pseudossignificância empírica é baseada em 999 permutações aleatória. Fonte: elaboração própria.

Com base na contiguidade do tipo torre (rook), a Tabela 2 apresenta os valores encontrados da estatística univariada e bivariada de $I$ de Moran das variáveis em estudo. A estatística $I$ de Moran univariada indica a presença de autocorrelação espacial positiva e estatisticamente significativa (em 1\%) para todas as variáveis em análise, isto é, as observações estão associadas com a sua localização no espaço, sofrendo interferências não somente da região em que se encontram, mas também das demais que as circundam.

Quanto ao I de Moran bivariado entre desmatamento e PIB per capita, o resultado aponta a presença de autocorrelação espacial positiva baixa entre essas duas variáveis, estatisticamente significativa em 5\%. Na análise entre o desmatamento e as variáveis explicativas adicionais, verifica-se a indicação de autocorrelação espacial negativa para as variáveis área plantada (AREAPLANT) e efetivo do rebanho (REBANHO), tendo em vista sua significância estatística. A exceção é a variável de demografia (DEMOG), uma vez que o p-valor aponta para sua não significância estatística. 
Tabela 2 - Estatística I de Moran das variáveis em estudo.

\begin{tabular}{|l|c|c|c|c|}
\hline Variável & $\begin{array}{c}I \text { de Moran } \\
\text { univariado }\end{array}$ & $\mathrm{p}$-valor* & $\begin{array}{c}I \text { de Moran } \\
\text { bivariado** }\end{array}$ & p-valor* \\
\hline DESMAT & 0,4361 & 0,0010 & & \\
\hline Y & 0,3067 & 0,0010 & 0,0801 & 0,0390 \\
\hline DEMOG & 0,4077 & 0,0010 & $-0,0782$ & 0,6150 \\
\hline AREAPLANT & 0,7432 & 0,0010 & $-0,1510$ & 0,0010 \\
\hline REBANHO & 0,7230 & 0,0010 & $-0,1746$ & 0,0010 \\
\hline
\end{tabular}

*A pseudossignificância empírica é baseada em 999 permutações aleatórias. ** I de Moran bivariado entre a variável dependente DESMAT e as variáveis explicativas.

Fonte: Elaboração própria.

O Mapa 1 apresenta a detecção de outliers para a área desmatada dos municípios do Cerrado em 2008, com base na ferramenta boxmap, considerando um hinge igual a 3.0. Foram identificados 61 municípios considerados outliers superiores:22 estão no Estado de Mato Grosso; 12, no Maranhão; 7, na Bahia; 5, no Mato Grosso do Sul; 5, no Tocantins; 4, no Piauí; 3, em Goiás; e 3, em Minas Gerais. Nenhum município da amostra apresentou-se como outlier inferior. 


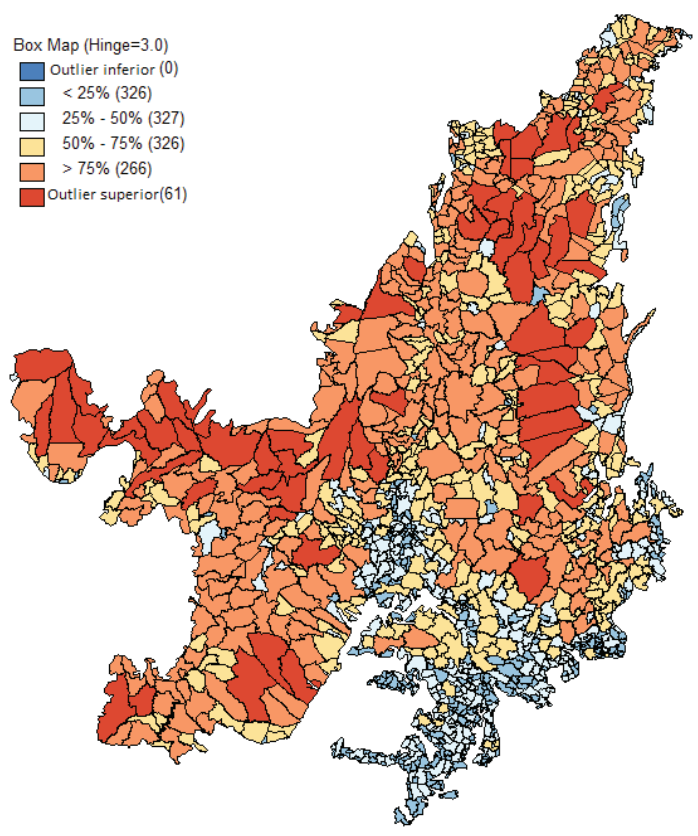

Mapa 1 - Boxmap do desmatamento em 2008.

Fonte: Elaboração própria.

O Mapa 2 apresenta os padrões de aglomeração local, denominados clusters, e, ao lado, o mapa de significância multivariados para o desmatamento e PIB per capita no Cerrado no ano de 2008. São percebidos, no mapa, destacados em cinza escuro, bolsões de forte desmatamento, caracterizados pelo padrão de associação denominado Alto-Alto, indicando que municípios com alto PIB per capita são circundados por municípios com alto desmatamento. Possivelmente tais regiões tenham recebido destaque na análise do desmatamento do período por serem novas regiões de expansão da fronteira agrícola, como, à esquerda, o estado de Mato Grosso, e, à direita, o oeste do estado da Bahia e o sul do Piauí. 

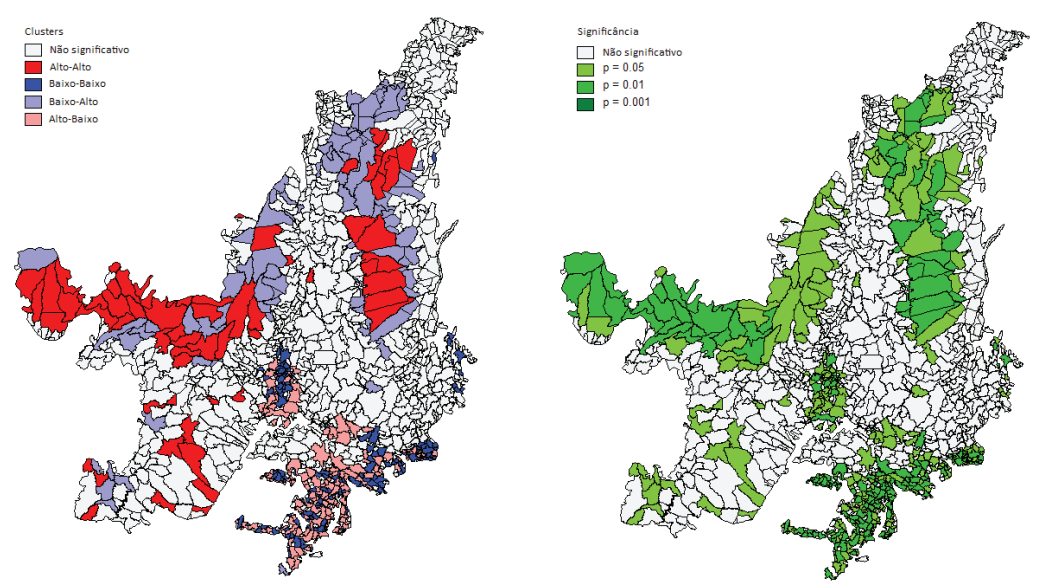

Mapa 2 - Mapa de cluster espacial multivariado e mapa de significância LISA, do desmatamento e PIB per capita dos municípios do Cerrado em 2008.

Fonte: Elaboração própria.

Estimação e análise dos modelos econométricos

Estimou-se primeiramente o modelo clássico de regressão linear por MQO, sem correção para dependência espacial. Testou-se a hipótese de ausência de autocorrelação espacial e verificou-se que os testes Multiplicador de Lagrange para defasagem $\left(\mathrm{ML}_{\rho}\right)$ e Multiplicador de Lagrange para o erro $\left(\mathrm{ML}_{\lambda}\right)$ eram estatisticamente significativos. Sendo assim, há indicação de presença de autocorrelação espacial, confirmando a necessidade de se optar por modelos que considerem tal efeito espacial.

A versão robusta dos testes de Multiplicador de Lagrange para defasagem $\left(\mathrm{ML}_{\rho}\right)$ e de Multiplicador de Lagrange para o erro $\left(\mathrm{ML}_{\lambda}\right)$ apontam que o modelo mais indicado seria o de defasagem espacial. De qualquer forma, os cinco modelos espaciais propostos foram estimados e os resultados obtidos foram comparados. Os resultados encontrados estão apresentados no Quadro 2. 
Quadro 2 - Resultado dos modelos estimados com correção para dependência espacial.

\begin{tabular}{|l|l|l|l|l|l|}
\hline Variável & modelo $(1)$ & modelo $(2)$ & modelo $(3)$ & modelo $(4)$ & modelo (5) \\
\hline$\rho$ & $0,492^{*}[0,302]$ & & & ns & \\
\hline$\lambda$ & & $0,503^{*}[0,03]$ & & & ns \\
\hline Constante & $30,539^{*}[7,046]$ & $67,373^{*}[10,69]$ & $18,153^{*}[2,015]$ & $18,593^{*}[2,28]$ & $18,239^{*}[2,013]$ \\
\hline $\mathrm{Y}$ & $3,616^{*}[0,534]$ & $3,650^{*}[0,61]$ & $0,147^{*}[0,031]$ & $0,148^{*}[0,03]$ & $0,147^{*}[0,030]$ \\
\hline Y2 & $-0,020^{*}[0,004]$ & $-0,021^{*}[0,005]$ & $0,306^{*}[0,033]$ & $0,306^{*}[0,033]$ & $0,305^{*}[0,033]$ \\
\hline DEMOG & $-0,026^{* *}[0,011]$ & $\mathrm{ns}$ & $0,106^{*}[0,029]$ & $0,107^{*}[0,029]$ & $0,106^{*}[0,029]$ \\
\hline AREAPLANT & $-90,051^{*}[15,33]$ & $-99,16^{*}[21,82]$ & $0,126^{*}[0,029]$ & $0,126^{*}[0,029]$ & $0,126^{*}[0,029]$ \\
\hline REBANHO & $-0,5802^{*}[0,096]$ & $-0,786^{*}[0,14]$ & $0,082^{*}[0,026]$ & $0,0828[0,026]$ & $0,081^{*}[0,026]$ \\
\hline WY & & & $-0,838^{*}[0,125]$ & $-0,858^{*}[0,135]$ & $-0,845^{*}[0,125]$ \\
\hline WY2 & & & $-0,005^{*}[0,001]$ & $-0,0007^{*}[0,001]$ & $-0,005^{*}[0,001]$ \\
\hline WDEMOG & & & $n s$ & $n s$ & $n s$ \\
\hline WAREAPLANT & & & $-0,031^{*}[0,006]$ & $-0,030^{*}[0,006]$ & $-0,031^{*}[0,006]$ \\
\hline WREBANHO & & & $-0,291^{* *}[0,11]$ & $-0,29^{*}[0,118]$ & $-0,29^{* *}[0,118]$ \\
\hline
\end{tabular}

Fonte: Elaboração própria.

Notas: (1) modelo de defasagem espacial - SAR, (2) modelo de erro espacial SEM, (3) modelo regressivo cruzado espacial, (4) modelo Durbin espacial, (5) modelo Durbin de erro espacial.

$*$ = coeficiente significativo a $1 \%$. $* *$ coeficiente significativo a $5 \%$. ns $=$ coeficiente não significativo em $5 \%$. [·] = erro padrão.

O modelo (1) de defasagem espacial (SAR) apresenta coeficientes significativos para todas as variáveis explicativas incluídas no modelo, inclusive para o coeficiente de defasagem espacial $\rho$. As variáveis explicativas adicionais DEMOG, AREAPLANT e REBANHO, apesar da significância dos coeficientes, apresentaram sinais inversos aos esperados. Já as variáveis explicativas básicas da hipótese da Curva de Kuznets Ambiental, o PIB per capita (Y) e sua forma quadrática (Y2), apresentaram os sinais esperados, configurando o formato de "U" invertido da CKA, indicando que o aumento do PIB per capita está relacionado com a ampliação do desmatamento, embora a tendência sofra inversão após o ponto de máximo da curva. 
O modelo (2) de erro espacial apresentou coeficientes estatisticamente significativos para as variáveis, com exceção do coeficiente da variável demografia DEMOG. O parâmetro de erro espacial $\lambda$ também se apresentou significativo e com sinal positivo. Os coeficientes das variáveis AREAPLANT e REBANHO apresentaram sinais inversos aos esperados. Os coeficientes das variáveis explicativas básicas da CKA, como PIB per capita e sua forma quadrática, além da significância estatística, apresentaram os sinais esperados, corroborando a hipótese da CKA. Apesar de bastante satisfatórios e semelhantes aos obtidos no modelo (1), o modelo (2) apresenta um valor maior para o critério de informação de Akaike (AIC), indicando uma ligeira piora em relação ao modelo anterior.

O modelo (3) apresentou significância estatística para todos os coeficientes das variáveis explicativas do modelo, como exceção do coeficiente da variável espacialmente defasada WDEMOG. As variáveis explicativas básicas do modelo não legitimam a hipótese da CKA, visto que a forma quadrática do PIB per capita apresenta sinal positivo, inverso ao esperado, configurando uma parábola com concavidade para cima. No modelo (4), os coeficientes das variáveis explicativas apresentaramse estatisticamente significativos, à exceção do coeficiente da variável espacialmente defasada WDEMOG. Nesse modelo, o coeficiente de defasagem espacial $\rho$ se mostrou não significativo estatisticamente. O modelo (5) exibiu os coeficientes das variáveis explicativas estatisticamente significativas, à exceção do coeficiente da variável espacialmente defasada WDEMOG. Nesse modelo, o parâmetro de erro espacial $\lambda$ apresentou-se estatisticamente não significativo.

Uma vez obtidos resultados que confirmaram a hipótese da curva em forma de "U" invertido, quando testado o modelo básico da CKA com a variável $\mathrm{Y}$ em sua forma quadrática, tanto no modelo de defasagem espacial quanto no modelo de erro espacial, optou-se por refazer tais testes incluindo a forma cúbica da variável renda per capita (Y3).

Mais uma vez evidenciada a necessidade de optar por modelos que considerem os efeitos espaciais, foram estimados novamente tanto 
os modelos de erro espacial (apontado pelo teste da robustez do Multiplicador de Lagrange) - Modelo ( $\left.1^{*}\right)$ - quanto o modelo de defasagem espacial - Modelo $(2 *)$, agora também com a forma cúbica da variável PIB per capita, visto que nos testes anteriores esses foram os modelos que confirmaram a hipótese da CKA. Os resultados encontramse disposto no Quadro 3.

Quadro 3 - Resultado dos modelos estimados com correção para dependência espacial, com variável adicional.

\begin{tabular}{|l|l|l|}
\hline Variável & modelo $\left(1^{*}\right)$ & modelo $(2 *)$ \\
\hline$\rho$ & $0,248^{*}[0,024]$ & \\
\hline$\lambda$ & & $0,398^{*}[0,034]$ \\
\hline constante & ns & $648,01^{*}[119,302]$ \\
\hline $\mathrm{Y}$ & $74,541^{*}[10,498]$ & $53,858^{*}[11,629]$ \\
\hline Y2 & $-1,273^{*}[0,221]$ & $-0,856^{*}[0,233]$ \\
\hline Y3 & $0,004^{*}[0,001]$ & $0,003^{*}[0,001]$ \\
\hline DEMOG & ns & ns \\
\hline AREAPLANT & $-812,04^{*}[186,295]$ & $-922,56^{*}[229,982]$ \\
\hline REBANHO & $-3,82^{*}[1,165]$ & $-6,83^{*}[1,486]$ \\
\hline
\end{tabular}

Fonte: Elaboração própria.

Notas: (1) modelo de defasagem espacial - SAR, (2) modelo de erro espacial - SEM.

$*=$ coeficiente significativo a $1 \%$. $* *$ coeficiente significativo a $5 \%$. ns $=$ coeficiente não significativo em $5 \%$. ['] = erro padrão.

Tanto o modelo de erro espacial $\left(1^{*}\right)$ quanto o modelo de defasagem espacial $\left(2^{*}\right)$ apresentam coeficientes das variáveis explicativas básicas significativos estatisticamente e os sinais esperados condizentes com a hipótese, configurando assim uma curva em forma de "N". Infere-se dessa análise que o desmatamento no Cerrado, nas condições analisadas, torna a crescer conforme o PIB per capita aumenta. Deste modo, o crescimento econômico não é solução para o desmatamento. Esse resultado implica 
haver espaço para a realização de política pública no sentido de reduzir o desmatamento no Cerrado.

\section{Conclusão}

O presente estudo verificou a existência de relação entre o desmatamento ocorrido no bioma Cerrado no ano de 2008 e o nível de atividade econômica na região, sob a hipótese da Curva de Kuznets Ambiental. Para tanto, definiu-se como variável dependente do modelo testado a área desmatada de Cerrado dos municípios e como variáveis explicativas o PIB per capita e suas formas quadrática e cúbica, a densidade demográfica, a área plantada e o efetivo dos rebanhos bovinos. O tratamento estatístico dos dados se deu sob a forma de uma cross-section, tendo em vista a indisponibilidade de dados referentes ao desmatamento dos municípios que constituem o Cerrado, para outros períodos.

Visto que a localização geográfica é um fator bastante relevante para a economia ambiental, optou-se por considerar os efeitos espaciais. Construiu-se o mapa do bioma Cerrado a partir do mapa do Brasil disponibilizado pelo IBGE. Metodologicamente, foi feita a análise exploratória de dados espaciais e, posteriormente, estimou-se o modelo pelo método de mínimos quadrados ordinários (sem efeitos espaciais) e modelos com efeitos espaciais como modelo de defasagem espacial, modelo de erro, modelo Durbin espacial (de defasagem e do erro) e modelo regressivo cruzado espacial.

Primeiramente, estimou-se o modelo proposto utilizando somente a forma quadrática do PIB per capita e as demais variáveis explicativas adicionais. Os resultados corroboram a hipótese do "U" invertido para os modelos de defasagem espacial e de erro espacial, indicando inicialmente aumento do desmatamento associado ao crescimento da renda e, posteriormente, inversão dessa tendência: após o ponto de máximo, a continuidade do aumento da renda contribui para a redução da degradação. Os demais modelos (regressivo cruzado espacial, Durbin espacial e Durbin espacial do erro) apontam para uma relação linear crescente entre o PIB per capita 
ao quadrado e o desmatamento, mas sem configurar hipótese da CKA.

Uma vez analisados tais resultados, incluiu-se a forma cúbica da variável PIB per capita, a fim de verificar se, após o ponto que expressa a queda da degradação, o crescimento continuado da renda contribuiria para o desmatamento tornar a crescer. Optou-se por refazer as estimações somente para os modelos de defasagem espacial e de erro espacial, uma vez que só ambos corroboraram a hipótese proposta do " $U$ " invertido. Os resultados encontrados apontam que, a princípio, o crescimento da renda per capita ajuda a diminuir o desmatamento na região, mas que, posteriormente, o desmatamento torna a crescer, configurando assim uma curva em forma de "N", e não mais a curva CKA em "U" invertido.

A interpretação deste resultado é que o crescimento econômico não é suficiente para reduzir o desmatamento depois de certo nível de renda. Pelo contrário, numa relação na forma de "N", o crescimento econômico agrava o desmatamento do bioma Cerrado. Em consequência, conclui-se que é necessário implementar políticas públicas adequadas para induzir a diminuição da degradação ambiental no Cerrado brasileiro.

A escassez de dados para região, principalmente variáveis ambientais, foi fator bastante limitador ao estudo. Assim, para trabalhos futuros, à medida que mais dados estejam disponíveis, sugere-se ampliar a análise, podendo ser feita sob a forma de dados em painel a fim de controlar as características municipais não observadas, invariantes no tempo.

\section{Referências}

ALMEIDA, E. S. Econometria Espacial Aplicada. $1^{\text {a }}$ ed. Campinas: Alínea Editora, 2012.

ANSELIN, L. Local indicators of spatial association - LISA. Geographical Analysis, 27, n. 2, p. 93-115, 1995. 
ANSELIN, L. Spatial Econometrics. USA: University of Texas, 1999.

CARVALHO, T. S.; ALMEIDA, E. A hipótese da Curva de Kuznets Ambiental Global: uma perspectiva econométrico-espacial. Est. Econ., São Paulo, v. 40, n 3, p. 587-615, jul.-set. 2010.

EMBRAPA CERRADOS. IV Plano Diretor da Embrapa Cerrados 2008 2011 - 2023 / Embrapa Cerrados - Planaltina. DF: Embrapa Cerrados, 2008.

GROSSMAN, G. M.; KRUEGER, A. B.. Environmental Impacts of a North American Free Trade Agreement. USA: National Bureau of Economic Research, NBER Working Paper nº 3914, nov. 1991.

IBAMA - Instituto Brasileiro do Meio Ambiente e dos Recursos Naturais Renováveis. Relatório técnico de monitoramento do desmatamento no Bioma Cerrado, 2002 a 2008: dados revisados. Acordo de cooperação técnica MMA/ IBAMA/PNUD. Brasília: Centro de Sensoriamento Remoto - CSR/IBAMA, nov. de 2009. Disponível em:

http://siscom.ibama.gov.br/monitorabiomas/cerrado/RELATORIO_ CERRADO_2008-2009.pdf .

LESAGE, J. R. Spatial Econometrics. USA: University of Toledo, 1998.

LESAGE, J. P. e PACE, R. K. Introduction to Spatial Econometrics. CRC Press, Boca Raton, 2009.

MCPHERSON, M. A.; NIESWIADOMY, M. L. Environmental Kuznets Curve: Threatened Species and Spatial Effects. Ecological Economics, v. 55, p. 395-407, 2005.

MMA-MINISTÉRIO DO MEIO AMBIENTE. Projeto de Monitoramento do Desmatamento dos Biomas Brasileiros por Satélite (PMDBBS). Disponível em: http://siscom.ibama.gov.br/monitorabiomas/. Acesso em: 8 de março de 2011.

MUELLER, C. C. Os economistas e as relaçães entre o sistema econômico e o meio ambiente. Brasília: Ed. UnB, 2007. p. 60-85. 
OLIVEIRA, R. C. Curva de Kuznets Ambiental para a Amazônia Legal. 2009. 160 f. Dissertação (Mestrado em Economia Aplicada), Universidade Federal de Juiz de Fora, Juiz de Fora. 2009

ROTHMAN, D. S.; de BRUYN, S. M. Probing into the environmental Kuznets curve hypothesis. Ecological Economics, v. 25, p. 143-145, 1998. 
REVISTA DE ECONOMIA E AGRONEGÓCIO, VOL.10, $N^{\circ} 3$ 